\title{
METODOLOGÍA PBLE COMO GUÍA DEL PROCESO DE APRENDIZAJE EN INGENIERÍA. PRIMEROS PASOS EN LA UR.
}

\author{
José Antonio Gómez Cristóbal \\ Joaquín Ordieres Meré \\ Manuel María Ruiz de Adana Santiago \\ Universidad de La Rioja
}

\begin{abstract}
RESUMEN: En este trabajo se dan a conocer algunas de las actuaciones que un equipo del Departamento de Ingeniería Mecánica de la Universidad de La Rioja desarrolla a modo de especificación de diseño conceptual del método formativo. Constituye una propuesta de implantación, que incluirá un plan de alcance, de comunicación y de riesgos, de cara a proponer a los usuarios de la Universidad un método alternativo y algo más acorde con las tendencias europeas que en este sentido se van produciendo desde hace ya algunos años. La aproximación seleccionada para la propuesta de implantación es la basada en la metodología PBLE (Project Based Learning Engineering).
\end{abstract}

ABSTRACT: This work presents some of the actions that a team of the Mechanical Engineering Department of the University of La Rioja develops as conceptual design specification of the formative method. It is a proposal of implantation, which will include a plan of communication and risks, to propose to the University users an alternative method in agreement with the European tendencies that go away in this sense during last years. The selected approximation for the proposal of implantation is based in the PBLE (Project Based Learning Engineering) methodology.

PALABRAS CLAVE: Aprendizaje, innovación docente, docencia de la ingeniería, PBL, PBLE. PBLE.

KEYWORDS: Learning, teaching innovation, engineering teaching, $\mathrm{PBL}$,

\section{INTRODUCCIÓN}

Actualmente las universidades españolas, en general, y las escuelas de ingeniería, en particular, están inmersas en un periodo de revisión de planes docentes para su adecuación al crédito ECTS. 
Tomando como punto de partida los actuales defectos y carencias detectados tanto en los planes de estudio actuales como en las metodologías docentes aplicadas, se pretende formalizar una propuesta alternativa que podría inspirar una nueva orientación hacia el concepto de ciclo de vida, tanto del egresado como del alumno.

En este trabajo se lleva a cabo una breve descripción de los principales cambios que, a nivel de propuesta, el equipo de trabajo del Departamento de Ingeniería Mecánica de la Universidad de La Rioja viene diseñando en los últimos meses, que no es sino un caso específico de la metodología PBLE.

En la primera parte del trabajo se analizan diversas estrategias docentes para, partiendo de su comparación, pasar posteriormente, en la segunda parte del trabajo, a proponer el ciclo de vida del paso por la Universidad del alumno en el marco del Espacio Europeo de Educación Superior (EEES), con una similitud clara con el ciclo de vida del profesional en su contexto futuro. Con este espíritu se propone, en la tercera parte del trabajo, una metodología formativa en las titulaciones cuya docencia es responsabilidad del Departamento de Ingeniería Mecánica de la Universidad de la Rioja. Por último, en la última parte del trabajo, se proponen unas conclusiones a la vista del trabajo que está siendo realizado.

\section{ESTRATEGIAS DOCENTES}

Pueden emplearse distintas estrategias a la hora de abordar la enseñanza universitaria de las ingenierías. En este apartado se discuten algunas de ellas así como sus ventajas e inconvenientes. No se pretende ser exhaustivo en este apartado, sino hacer un compendio que, en términos de grandes familias de técnicas formativas, permita comparar los grandes enfoques y sus diferencias, pues se propone una adaptación de una de ellas como aportación para la formación en Ingeniería.

\subsection{Aprendizaje basado en el profesor, TCL (Teacher Center Learning)}

El TCL ha sido el método tradicionalmente empleado en la docencia en ingeniería. Este método, considerado por muchos como ideal, está, sin embargo, limitado en algunos aspectos ya que no proporciona a los estudiantes oportunidades para ser creativos ni responsables. En general, los sistemas de aprendizaje tradicionales tratan de resolver problemas con una solución única y bien definida. Los estudiantes perciben al profesor como una persona de confianza y experimentada de la que pueden aprender. Sin embargo, el éxito de los estudiantes depende de la capacidad del profesor para enseñar y motivar a los estudiantes. En casos extremos los estudiantes pueden incluso abandonar el curso.

Una variante de esta metodología es la que tradicionalmente se emplea en la Universidad privada española, que es complementada mediante un seguimiento individualizado del trabajo de cada estudiante, de modo que se combina el TCL con metodologías de evaluación continuada o, al menos, de seguimiento continuado y planificación a corto plazo.

\subsection{Aprendizaje basado en el problema, PBL (Problem Based Learning)}

El volumen de conocimientos en cada área crece continuamente y la velocidad a la que crece también está aumentando. Los estudiantes no pueden aprender todo el 
material, pero pueden aprender cómo adquirir los conocimientos necesarios. Con la metodología PBL, los estudiantes aprenden a ser aprendices autodirigidos, independientes e interdependientes.

En un curso organizado con la metodología PBL los estudiantes forman pequeños grupos con un tutor para discutir un problema propuesto. Inicialmente los estudiantes exploran el problema usando sus conocimientos previos y su experiencia. Entonces analizan el problema y formulan hipótesis que pueden explicar el problema. Esta información es empleada por los alumnos para determinar qué información adicional necesitan para comprender y resolver el problema (Duch, 1995).

Los estudiantes investigan independientemente la información necesaria que permita confirmar o no las hipótesis planteadas. Esta nueva información se presenta al grupo y se valora en común la información proporcionada por cada miembro (Delisle, 1997). Esto puede conducir a:

- Una nueva formulación del problema

- La solicitud de información adicional al tutor

- La identificación de preguntas e información necesaria para distinguir entre las hipótesis y las explicaciones.

En el proceso el tutor actúa facilitando el trabajo más que enseñando. En vez de proporcionar respuestas el tutor plantea preguntas útiles y si es necesario proporciona la estructura de resolución del problema (Hoffman y Ritchie, 1997).

Finalmente los estudiantes y el tutor valoran la calidad de las respuestas obtenidas y la efectividad del proceso empleado, terminando con una reflexión del problema y un proceso de autoevaluación del alumno (Stepien y Gallagher, 1993).
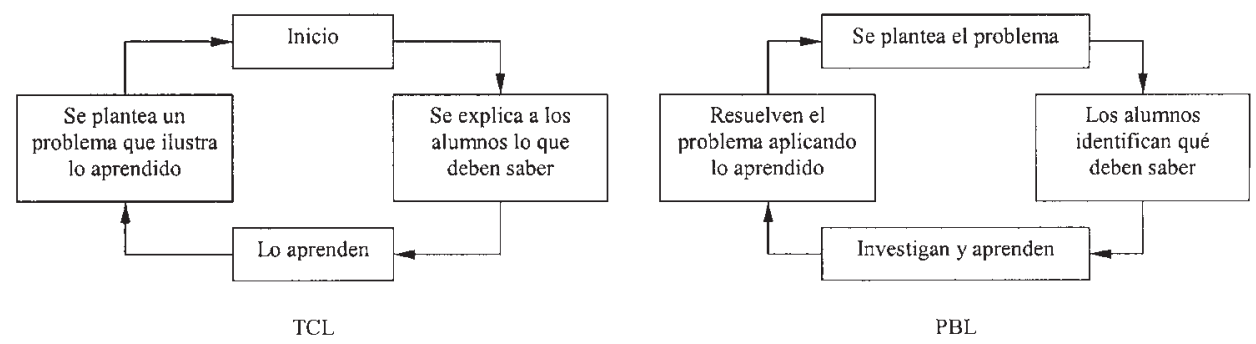

Figura 1. Procesos de aprendizaje TCL y PBL.

Por lo tanto la metodología PBL desarrolla una enseñanza cimentada sobre los siguientes principios básicos:

- Los problemas se diseñan para emular problemas del mundo real.

- Los problemas son complejos y cubren múltiples objetivos.

- El problema es introducido primero, antes de que el aprendizaje tenga lugar.

- Los procesos de aprendizaje, hechos y conceptos ocurren en el contexto de buscar una solución al problema. 
- Los procesos específicos o algoritmos son aprendidos según se necesiten.

- La estructura adicional para el aprendizaje es proporcional al nivel de experiencia del alumno.

- Gran parte de la estructura de aprendizaje se adquiere a través de cuestiones planteadas por el instructor.

- Los estudiantes que emplean este proceso trabajan en equipos o grupos para adquirir múltiples perspectivas sobre las posibles soluciones.

- La evaluación es coherente con el desarrollo del proceso de resolución del problema, balanceando el peso de los conocimientos teóricos con la actitud en la resolución del problema y con la marcha del proceso de aprendizaje.

\subsection{Aprendizaje basado en el proyecto en ingeniería, PBLE (Project Based Learning Engineering)}

La estrategia PBL resulta muy interesante desde el punto de vista del proceso de aprendizaje del alumno, y sin duda constituye una buena preparación para enfrentarse a los problemas del mundo real en muchos ámbitos profesionales.

Sin embargo, en el ámbito de la ingeniería los problemas a resolver casi nunca son problemas concretos sino que en muchos casos los proyectos de ingeniería engloban un conjunto de problemas interrelacionados que el ingeniero debe resolver. Por lo tanto la metodología a emplear en el proceso de aprendizaje debe reconducirse para que el proceso de formación recoja las complejidades con las que el ingeniero se va a enfrentar en su etapa profesional. El PBLE cubre estos objetivos de formación ya que la producción de un producto final es el centro del proceso de aprendizaje.

La metodología PBLE sigue el enfoque del PBL. Ambas son estrategias de aprendizaje centradas en el alumno, sitúan al profesor en el rol de facilitador del proceso, se orientan a conectar a los estudiantes con tareas del mundo real para mejorar su aprendizaje, los alumnos trabajan en grupos cooperativos durante un tiempo extenso y de esta manera son capaces de buscar, encontrar, codificar y utilizar información procedente de múltiples fuentes (Woods, 1996).

En la metodología PBLE el producto final es el centro del proyecto. El producto final puede ser una obra civil, un equipo, una instalación, un sistema o similares. El nivel de tecnología y complejidad puede variar según el alcance del mismo, el objetivo de aprendizaje y el tiempo disponible en los créditos asignados al proyecto. Los productos finales:

- Son productos elaborados y constituyen en sí el proceso de producción

- Conducen el proceso de planificación, producción y evaluación

- Están constituidos por distintos problemas que requieren el desarrollo de las habilidades de los alumnos para su resolución

El enfoque PBLE emplea un modelo de producción cuyas características más significativas se citan a continuación (Banks, 1997; Blumenfeld et al., 1991).

- Los estudiantes definen el propósito para crear el producto final e identifican su audiencia.

- Los estudiantes investigan sus temas, diseñan su producto y crean un plan para la dirección del proyecto. 
- Los estudiantes comienzan el proyecto, resuelven los problemas y cuestiones que surgen en la producción, y terminan su producto.

- Los estudiantes presentan el producto que han creado.

- Los estudiantes reflexionan y evalúan su propio trabajo.

El proceso completo refleja actividades de producción del mundo real y se utilizan las ideas propias y aproximaciones de los estudiantes para acompañar las tareas. Aunque el producto final es el centro del PBLE, lo importante es el conocimien to y las habilidades adquiridas por los estudiantes durante el proceso de producción.

\section{VENTAJAS E INCONVENIENTES DEL PBLE: DISCUSIÓN}

Tanto el PBL como el PBLE incorporan muchas de las prácticas consideradas la desiderata del buen docente: está centrado en el alumno, fomenta la motivación intrínseca, promueve el aprendizaje activo y profundo, utiliza el conocimiento previo de los alumnos, anima a la crítica del proceso enseñanza-aprendizaje, desarrolla habilidades aprendidas en etapas preuniversitarias, conduce a un currículo orientado a la investigación, implica retroalimentación y conduce a la autovaloración del alumno.

Como avalan numerosas investigaciones (Woods, 1994) plantear un proyecto concreto como foco de la adquisición del conocimiento ayuda a los estudiantes a retener su aprendizaje y comprenderlo mejor. Los estudiantes desarrollan habilidades dentro de un contexto, lo que permite mejorar su propio conocimiento y adquirir nuevas habilidades en el terreno interpersonal. De esta manera los estudiantes se enriquecen adquiriendo un conjunto de habilidades especialmente valiosas en su vida postuniversitaria.

Finalmente el PBLE ofrece a los profesores la oportunidad para desarrollar a los estudiantes que desean: independientes, auto motivados, hábiles en un amplio conjunto de disciplinas, desafiando los parámetros del curso y produciendo trabajo interesante. Estos son los estudiantes que no sólo trabajan para aprobar el examen, sino que desean comprender y aprender.

Hay un coste cierto tanto para profesores y alumnos al emplear el enfoque PBLE. Un coste indirecto es el shock inicial ya que la mayoría de las estrategias de enseñanza-aprendizaje tradicionales son inadecuadas en el entorno PBLE. Para el docente el cambio de rol de experto profesor a facilitador del proceso de enseñanza-aprendizaje no es fácil. A su vez el alumno manifiesta reticencias ante el cambio a un sistema nuevo y desconocido.

Una preparación adecuada es esencial tanto para los docentes como para los alumnos. Con todo, pueden surgir problemas al principio, por lo que la gestión del tiempo pasa a ser un elemento clave.

Sin apoyo a nivel departamental o de Vicerrectorado el esfuerzo para realizar el cambio puede ser difícil de sostener. El profesorado necesita apoyo moral para ocuparse del cambio que representa la implantación del PBLE. También es importante compartir experiencias con colegas expertos en entornos PBLE similares.

En PBLE el profesor incide más en el proceso de aprendizaje de los alumnos que en el aseguramiento de la revisión o impartición de unos determinados contenidos. Manteniendo el equilibrio entre el aprendizaje autodirigido de los alumnos y los 
objetivos de la titulación, los docentes deben aprender también a sentirse cómodos con unos resultados de aprendizaje menos predecibles.

El cambio docente en el método de enseñanza se basa en la oportunidad de los profesores para observar el impacto de su enseñanza en el aprendizaje de sus alumnos. La mayoría de los docentes no usa sus clases como laboratorios para el estudio del aprendizaje (Cross, 1991).

\section{VIABILIDAD DE LA METODOLOGÍA PBLE EN INGENIERÍA}

A pesar de los inconvenientes mencionados anteriormente el PBLE se perfila como uno de los principales desarrollos a nivel de la enseñanza universitaria de los últimos tiempos (McKeachie y Gibbs, 1999). Prueba de ello es el hecho que algunas escuelas de ingeniería han apostado por este sistema y los resultados de todas estas experiencias parecen en su conjunto prometedores.

El objetivo principal de la labor académica es revisar la presente situación e introducir los cambios necesarios para mejorar el proceso de educación (Angelo y Cross, 1993; Mehta y Nem, 1998). El PBLE mejora habilidades importantes tales como analizar y resolver proyectos, encontrar, evaluar y usar recursos adecuados de aprendizaje, trabajar en equipo y comunicarse efectivamente, tanto de forma verbal como por escrito.

Asumiendo el esfuerzo en el ámbito institucional, docente y de los alumnos para realizar el cambio en la estrategia del proceso enseñanza-aprendizaje mediante PBLE, los resultados alcanzables mediante esta estrategia, que se enumeran a continuación, son muy deseables:

- Dado que el aprendizaje ocurre en un entorno similar al entorno laboral, las habilidades adquiridas para resolver problemas serán más fácilmente transferibles al entorno de trabajo.

- Los estudiantes no aprenden hechos, habilidades y conceptos como entidades separadas; en cambio pueden interconectarlos para resolver problemas reales.

- Los estudiantes aprenden a encontrar, evaluar y usar adecuadamente los recursos de aprendizaje.

- Los estudiantes mejoran su habilidad para trabajar en equipo.

- Los estudiantes aumentan sus habilidades de comunicación explicando los resultados de su investigación a los miembros del equipo y presentando un informe final.

Aunque la estrategia PBLE puede aplicarse en principio a cualquier plan de estudios, en el caso de la ingeniería resulta conveniente diseñar adecuadamente las etapas formativas con objeto de que el aprendizaje en las habilidades técnicas, organizativas, toma de decisiones, liderazgo, etc, sean equilibradas y el resultado sea un ingeniero equilibrado y flexible dispuesto a integrarse rápidamente y con éxito en el entorno laboral. En efecto, independientemente de la metodología de transmisión de conocimientos el rol que juega el alumno en el sistema clásico durante su paso por la Universidad es siempre el mismo (ver metodologías de aprendizaje TCL en los apartados anteriores), a diferencia del ciclo que desarrolla en su vida profesional, en la que la adquisición de experiencia y, por tanto, de habilidades le van permitiendo desempeñar diferentes papeles. 
En este caso, en el trabajo que se desarrolla en la Universidad de La Rioja, se pretende que el alumno perciba ese cambio de rol según va adquiriendo mayor grado de experiencia en la titulación, y que le sirva también para entender como natural la posterior evolución de su vida profesional. Esta innovación permitirá también una visión más integral del proceso formativo.

La estructura del plan de estudios debe facilitar que el alumno asuma los distintos roles que el futuro ingeniero va a tener en su ámbito profesional. Este cambio de rol puede adecuarse fácilmente al plan de estudio de grado en ingeniería de 4 años previsto a partir de la declaración de Bolonia. La equivalencia entre las etapas profesionales y el desarrollo del calendario académico a 4 años se muestra en la figura adjunta.

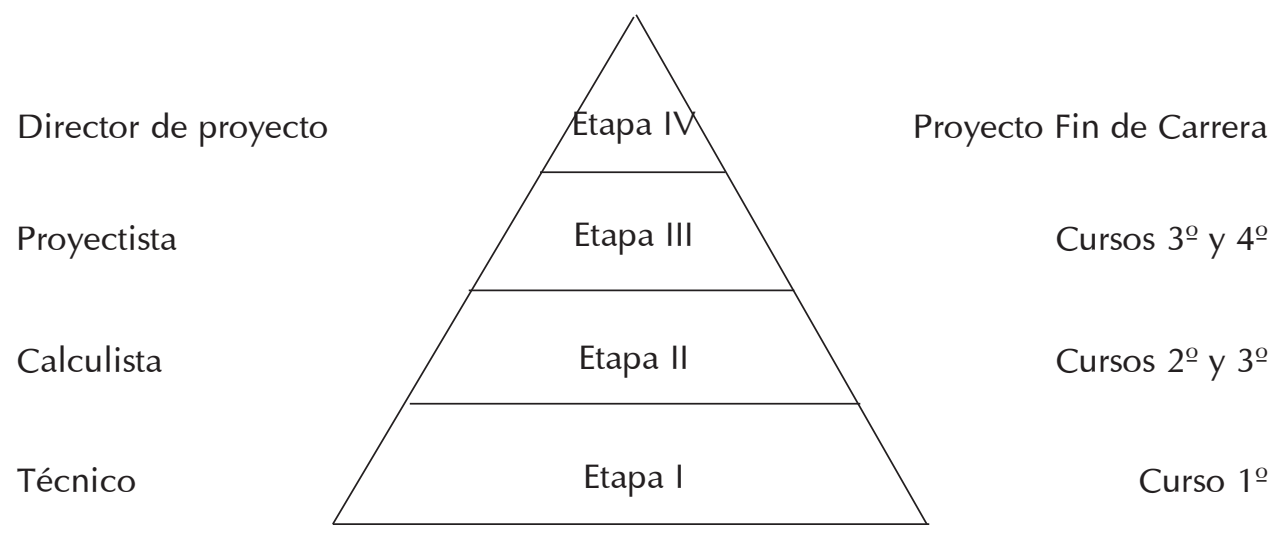

Figura 2. Equivalencia entre etapas de aprendizaje y calendario académico.

En el modelo propuesto el alumno se implica cada curso en un nuevo proyecto, integrándose en un equipo formado por alumnos de todos los cursos y donde cada uno asume un rol y unas determinadas funciones de acuerdo a los conocimientos y a las actividades que debe trabajar durante el curso académico. Los alumnos de primer curso, centrados en asignaturas de carácter científico básico, desempeñan un rol básicamente técnico, ocupándose de las tareas más sencillas, como la revisión de documentación técnica, especificaciones de materiales, tareas de diseño asistido por ordenador, etc. Un segundo rol, que desempeñan los alumnos de $2^{\circ}$ y $3^{\circ}$ curso, centrados en materias con un fuerte contenido de cálculo, es el de calculista. El tercer rol, desempeñado por alumnos de $3^{\circ}$ y $4^{\circ}$ curso, y ya formados en materias más descriptivas orientadas a la realización de proyectos, es el de proyectista. Y por último, los alumnos matriculados en el Proyecto de Fin de Carrera, desempeñan el papel de director de proyecto, coordinando el trabajo de todos los miembros del equipo, gestionando los tiempos y los recursos y liderando el proyecto. Todos los miembros del equipo trabajan según un esquema colaborativo jerárquico, donde las decisiones tomadas por los alumnos más expertos condicionan y orientan el trabajo de los más inexpertos. 
A la vista de las cuestiones planteadas, la implantación del método conllevará las siguientes mejoras:

- Rápida integración del alumno al entorno universitario.

- Reducción de las tasas de fracaso académico.

- Mayor experiencia en la gestión de proyectos.

- Mejora de la comunicación de ideas y resultados.

- Rápida adaptación a diferentes entornos de trabajo.

- Mayor facilidad de aplicación de los conceptos a casos reales.

- Aumento de la responsabilidad del alumno sobre su formación.

El proceso de implantación de este sistema de aprendizaje mediante PBLE basado en el ciclo de vida laboral del ingeniero en la estructura académica propuesta de un título de grado de 4 años debe realizarse de manera planificada y de acuerdo con una serie de directrices fundamentales. Una síntesis del proceso de implantación se recoge a continuación según un esquema en seis fases. Las distintas fases no requieren necesariamente ser desarrolladas en el orden en que se citan y, en muchos casos, su desarrollo se solapa con otras fases.

\section{Fase I: Definición de los resultados de aprendizaje}

El diseño de cualquier estrategia docente debe partir de los resultados de aprendizaje que se deseen alcanzar (Biggs, 2002). En nuestro caso estos resultados vienen dados por el tipo de ingeniero que se desee formar. Partiendo de las características básicas del ingeniero se establecen los resultados de aprendizaje deseables. Estos resultados se agrupan en torno a 5 grandes bloques:

\section{Conocimientos y comprensión}

- Conocimientos en métodos matemáticos relevantes y principios de la ingeniería mecánica

- Conocimientos específicos en disciplinas propias del perfil: principios y técnicas de diseño en ingeniería, máquinas y mecanismos, ingeniería térmica, ingeniería de fabricación, ciencia de materiales, mecánica del medio continuo, etc.

- Dirección y gestión en ingeniería

- Comprensión de las restricciones externas

- Comprensión de las responsabilidades profesionales y éticas

- Comprensión del impacto de las soluciones de ingeniería en la sociedad

\section{Habilidades intelectuales}

- Habilidad para resolver problemas en ingeniería y diseño de sistemas mediante pensamiento creativo e innovador

- Habilidad para aplicar herramientas matemáticas, científicas y tecnológicas

- Habilidad para interpretar datos numéricos, aplicación de métodos matemáticos y diseño de experimentos para obtener nuevos datos 
- Habilidad para mantener una base teórica que permita la introducción de nueva tecnología

- Habilidad para aplicar soluciones con juicio profesional, equilibrando aspectos de costes, beneficios, seguridad, calidad y medio ambiente

- Habilidad para manejar y dirigir riesgos

\section{Habilidades prácticas}

- Uso de un amplio conjunto de herramientas, técnicas y equipos apropiados para su disciplina específica

- Uso de equipos reales y de laboratorio para generar datos

- Búsqueda de información

- Habilidades básicas de organización y dirección de proyectos

- Desarrollar, promover y aplicar sistemas de trabajo seguros

\section{Habilidades generales transferibles}

- Comunicación efectiva escrita y oral

- Uso de tecnologías de la información

- Dirigir recursos y tiempo de manera efectiva

- Trabajo independiente y en equipo multidisciplinar

- Emprender aprendizaje continuo para el desarrollo profesional

\section{Cualidades}

- Creativo, particularmente en el proceso de diseño

- Analítico en la formulación y solución de problemas

- Innovador en la solución de problemas de ingeniería

- Auto motivado

- Con criterio e integridad profesional guardando especial respeto a los asuntos éticos

- Entusiasta, en la aplicación del conocimiento, comprensión y habilidades en pos de la práctica de ingeniería

La programación temporal de la metodología PBLE para los estudios de grado en ingeniería debe asegurar el desarrollo progresivo del conjunto de habilidades que se recoge en la tabla adjunta. 


\begin{tabular}{|l|c|c|c|c|}
\hline HABILIDAD & I & II & III & IV \\
\hline Trabajo en equipo multidisciplinar & $\checkmark$ & $\checkmark$ & $\checkmark$ & \\
\hline Responsabilidad e implicación & $\checkmark$ & $\checkmark$ & $\checkmark$ & \\
\hline Comunicación oral y escrita & & $\checkmark$ & $\checkmark$ & $\checkmark$ \\
\hline Organización & & $\checkmark$ & $\checkmark$ & $\checkmark$ \\
\hline Pensamiento crítico & & $\checkmark$ & $\checkmark$ & $\checkmark$ \\
\hline Toma de decisiones & & & $\checkmark$ & $\checkmark$ \\
\hline Creatividad & & & $\checkmark$ & $\checkmark$ \\
\hline Habilidades directivas & & & & $\checkmark$ \\
\hline Liderazgo & & & & $\checkmark$ \\
\hline
\end{tabular}

Tabla 1. Habilidades a desarrollar en cada etapa de aprendizaje.

Estos resultados de aprendizaje deseables se pueden alcanzar mediante el adecuado diseño de la metodología PBLE en combinación con métodos docentes ya conocidos. Algunas directrices se mencionan en los siguientes apartados.

\section{Fase II: Definición del tipo de proyecto y diseño del mismo}

Para aprovechar las oportunidades que se presentan en el sistema de aprendizaje mediante proyectos se debe identificar el tipo de proyecto que es más adecuado con los resultados que se esperan en el proceso de aprendizaje. Algunas de estas variantes se recogen a continuación:

- Proyecto individual/en grupo

- Proyecto cerrado/abierto

- Proyecto incremental/innovador

- Proyecto multidisciplinar

- Proyecto industrial con empresa

Cada tipo de proyecto presenta una serie de ventajas y desventajas que deben evaluarse en cada caso, en función de los resultados que se deseen obtener (Howard y Sharp, 1996; Lewis y Habeshaw, 1997). En cualquier caso, la planificación del proyecto por parte del docente debe promover distintas habilidades en el alumno para lo cual el docente debe:

- Preparar una guía de aprendizaje mediante proyectos que incluya indicaciones sobre planificación de proyectos, trabajo en equipo e informes escritos, entre otros

- Identificar recursos y materiales útiles, tales como libros, artículos científicos y técnicos, y material en soporte digital 
- Emplear soportes virtuales para el proyecto, incluyendo un enlace de información, documentación clave, foro de discusión y otros

- Realizar sesiones preparatorias perfilando las ideas claves que los estudiantes necesitarán emplear para dirigir y desarrollar el proyecto

- Ofrecer información específica a determinados estudiantes ante la solicitud/necesidad que puedan tener en determinadas habilidades

- Incorporar en los objetivos exigencias para que los alumnos reflexionen sobre el desarrollo de las distintas habilidades a adquirir

La labor docente se completa con el seguimiento, apoyo y evaluación como se recoge más abajo.

\section{Fase III: Soporte teórico: habilidades basadas en conocimientos}

Además de aprender nuevos conceptos en el desarrollo de un proyecto los alumnos deben aplicar la teoría que han aprendido en módulos previos. Por lo tanto, al especificar un proyecto los resultados teóricos basados en conocimientos previos tales como habilidades numéricas, y la aplicación y retención de conceptos y principios clave son los primeros en ser definidos.

La adecuada combinación del sistema tradicional de metodología docente con la metodología PBLE ha sido el factor clave para el éxito de muchas experiencias en PBLE (Albanese y Mitchell, 1993; Farnsworth, 1994; Qin y Johnson, 1995)

Las habilidades basadas en conocimientos pueden adquirirse mediante:

- Clases magistrales. Proporcionan al estudiante información relevante sobre el desarrollo del proyecto.

- Clases de apoyo. Se establecen discusiones dirigidas y estructuradas.

- Aprendizaje independiente. Los estudiantes necesitan previamente ser dirigidos para ser capaces de identificar fuentes de información, extraer información relevante, analizar e interpretar la información, usar la información para la resolución de problemas en el marco del proyecto, y aplicar la información en el proyecto. Los materiales pueden incluir soporte tipo texto, artículos en revistas especializadas, $C D$, audio, video e internet.

- Trabajo en grupo. La división de la tarea en pequeñas áreas requiere una comunicación efectiva dentro del grupo para asegurar que todos los estudiantes conocen el fundamento teórico del problema

\section{Fase IV: Soporte práctico: habilidades transferibles basadas en proceso}

Para desarrollar el proyecto adecuadamente los estudiantes necesitan aprender distintas habilidades de planificación, organización, trabajo en equipo y de comunicación (Cawley, 1997; Toohey, 1999) El docente debe promover el desarrollo de estas habilidades a la hora de diseñar y planificar el proceso de aprendizaje mediante PBLE recogiendo los siguientes aspectos:

- Planificación y organización

- Identificar qué se debe hacer

- Decidir quién hará qué 
- Valorando cuánto tiempo llevará

- Identificando los recursos disponibles

- Generando un plan de proyecto

- Monitorizando el progreso

- Planificando las tareas finales del proyecto

- Habilidades de trabajo en equipo

- Establecer reglas de grupo para que el trabajo sea efectivo

- Clarificar propósitos, objetivos y prioridades

- Comunicación efectiva

- Apoyo mutuo

- Entrega del trabajo a tiempo

- Revisar y evaluar el rendimiento

- Habilidades de comunicación formales

- Informe escrito

- Informe interno

- Informe final

- Presentaciones

- Demostraciones

\section{Fase V: Soporte y seguimiento a individuos y grupos. Tutorización}

Durante el desarrollo de los proyectos los alumnos deben desarrollan un gran número de habilidades y actitudes. Por este motivo el soporte docente debe ser más amplio y debe cubrir áreas como:

- Animar al trabajo efectivo en equipo

- Desarrollar habilidades para la resolución de problemas

- Asegurar una buena presentación y expresión oral

- Desarrollar habilidades de auto aprendizaje

- Desarrollar habilidades de información mediante nuevas tecnologías

El tutor del proyecto dispone de distintos mecanismos de soporte que se recogen en la tabla adjunta. 
METODOLOGÍA PBLE COMO GUÍA DEL PROCESO DE APRENDIZAJE EN INGENIERÍA. PRIMEROS PASOS EN LA UR.

\begin{tabular}{|l|l|}
\hline MECANISMO DE SOPORTE & VENTAJAS \\
\hline Materiales en soporte papel & $\begin{array}{l}\text { Anima al autoaprendizaje } \\
\text { Recurso reutilizable }\end{array}$ \\
\hline Clases/seminarios & $\begin{array}{l}\text { Permite apoyo detallado sobre habilidades/teoría } \\
\text { Respuesta inmediata }\end{array}$ \\
\hline Soporte en red & Bajo coste, fácil de actualizar y acceder \\
\hline Foro de discusión & Evita repetir respuestas a las mismas dudas \\
\hline Correo electrónico & Inmediato, fácil de usar, privado \\
\hline Monitorización de portafolios & $\begin{array}{l}\text { Identifica si los estudiantes han dirigidos sus } \\
\text { esfuerzos, y qué habilidades han desarrollado }\end{array}$ \\
\hline Realimentación de evaluación & $\begin{array}{l}\text { Esencial para mantener seguimiento a los } \\
\text { estudiantes, e identificar si requieren apoyo }\end{array}$ \\
\hline Reuniones de grupo & $\begin{array}{l}\text { Permiten a los estudiantes desarrollar habilidades } \\
\text { para el trabajo en equipo, guiarles con apoyo } \\
\text { adecuado e identificar problemas que requieren } \\
\text { intervención }\end{array}$ \\
\hline Reunión de supervisión & $\begin{array}{l}\text { Permite apoyo detallado, oportunidad sustancial } \\
\text { de realimentación }\end{array}$ \\
\hline Sesiones de reflexión & $\begin{array}{l}\text { Permite a los estudiantes reflejar su rendimiento } \\
\text { actual e identificar estrategias para mejorar }\end{array}$ \\
\hline
\end{tabular}

Tabla 2. Mecanismos de soporte del tutor del proyecto.

En otros casos el soporte puede realizarse por el propio estudiante, por los compañeros del grupo, o mediante herramientas de soporte en red.

\section{Fase VI: Evaluación}

La evaluación permite mejorar el aprendizaje, aumentar el desarrollo del curso y medir el rendimiento del estudiante. La tabla adjunta ilustra los resultados de evaluación bajo estas tres áreas. 


\begin{tabular}{|l|l|l|}
\hline $\begin{array}{l}\text { EVALUACIÓN Y } \\
\text { APRENDIZAJE }\end{array}$ & $\begin{array}{l}\text { EVALUACIÓN Y } \\
\text { DESARROLLO DEL } \\
\text { CURSO }\end{array}$ & $\begin{array}{l}\text { EVALUACIÓN Y } \\
\text { RENDIMIENTO DEL } \\
\text { ESTUDIANTE }\end{array}$ \\
\hline $\begin{array}{l}\text { Proporciona } \\
\text { realimentación al profesor } \\
\text { para ser capaces de } \\
\text { planear más efectivamente } \\
\begin{array}{l}\text { su aprendizaje y su } \\
\text { rendimiento }\end{array}\end{array}$ & $\begin{array}{l}\text { Proporciona } \\
\text { realimentación }\end{array}$ & $\begin{array}{l}\text { Proporciona una medida } \\
\text { del éxito alentando a los } \\
\text { estudiantes a reflexionar } \\
\text { en su rendimiento y cómo } \\
\text { alcanzar las expectativas } \\
\text { de evaluación }\end{array}$ \\
\hline $\begin{array}{l}\text { Identifica si los } \\
\text { estudiantes necesitan } \\
\text { desarrollar habilidades } \\
\text { y competencias }\end{array}$ & $\begin{array}{l}\text { Permite evaluación de } \\
\text { la eficiencia de la } \\
\text { enseñanza }\end{array}$ & $\begin{array}{l}\text { Clasifica a los estudiantes } \\
\text { permitiendo al tutor iden- } \\
\text { tificar habilidades de } \\
\text { grupo y desarrollar estrate- } \\
\text { gias para encontrar sus } \\
\text { necesidades individuales }\end{array}$ \\
\hline $\begin{array}{l}\text { Aumentan la motivación } \\
\text { de los estudiantes }\end{array}$ & $\begin{array}{l}\text { Promueve el desarrollo } \\
\text { del curso mediante la } \\
\text { revisión del currículo }\end{array}$ & $\begin{array}{l}\text { Permite a los estudiantes } \\
\text { progresión alentando un } \\
\text { sentido de propiedad en } \\
\text { su aprendizaje y } \\
\text { realización }\end{array}$ \\
\hline
\end{tabular}

Tabla 3. Resultados de evaluación.

Para determinar si el estudiante ha alcanzado resultados relevantes de aprendizaje, la metodología PBLE dispone de una amplia variedad de métodos. Cada método se puede emplear para evaluar un conjunto de habilidades. La tabla adjunta recoge los métodos de evaluación a emplear en la metodología PBLE así como la persona que actúa como evaluador en cada caso. 
METODOLOGÍA PBLE COMO GUÍA DEL PROCESO DE APRENDIZAJE EN INGENIERÍA. PRIMEROS PASOS EN LA UR.

\begin{tabular}{|c|c|c|}
\hline MÉTODO & HABILIDADES & EVALUADOR \\
\hline $\begin{array}{l}\text { Informe } \\
\text { escrito }\end{array}$ & $\begin{array}{l}\text { Conocimiento y aplicación de la teoría } \\
\text { Resolución de problemas } \\
\text { Investigación } \\
\text { Aprendizaje independiente } \\
\text { Redacción y edición }\end{array}$ & $\begin{array}{l}\text { Tutores } \\
\text { Asesores externos }\end{array}$ \\
\hline $\begin{array}{l}\text { Producto, } \\
\text { prototipo o } \\
\text { modelo }\end{array}$ & $\begin{array}{l}\text { Resolución de problemas } \\
\text { Diseño y fabricación }\end{array}$ & $\begin{array}{l}\text { Tutor } \\
\text { Compañeros } \\
\text { Ingeniero en prácticas }\end{array}$ \\
\hline $\begin{array}{l}\text { Prueba de } \\
\text { prestaciones } \\
\text { Prestaciones } \\
\text { del producto }\end{array}$ & Evaluación & Tutor \\
\hline $\begin{array}{l}\text { Diario de } \\
\text { aprendizaje }\end{array}$ & Aprendizaje independiente & $\begin{array}{l}\text { Tutor } \\
\text { Compañeros } \\
\text { Mentor }\end{array}$ \\
\hline Viva voz & $\begin{array}{l}\text { Comprensión } \\
\text { Comunicación } \\
\text { Razonamiento }\end{array}$ & $\begin{array}{l}\text { Tutor } \\
\text { Ingeniero en prácticas }\end{array}$ \\
\hline Presentación & $\begin{array}{l}\text { Conocimiento } \\
\text { Comprensión } \\
\text { Habilidad para estructurar información } \\
\text { Habilidades de comunicación }\end{array}$ & $\begin{array}{l}\text { Tutores } \\
\text { El propio alumno } \\
\text { Compañeros } \\
\text { Ingeniero en prácticas }\end{array}$ \\
\hline Póster & $\begin{array}{l}\text { Presentación } \\
\text { Comunicación } \\
\text { Conocimiento y aplicación de la teoría }\end{array}$ & $\begin{array}{l}\text { Tutores } \\
\text { Compañeros } \\
\text { Ingeniero en prácticas }\end{array}$ \\
\hline Portafolio & $\begin{array}{l}\text { Creatividad } \\
\text { Habilidades de diseño asistidas por } \\
\text { ordenador }\end{array}$ & $\begin{array}{l}\text { Tutores } \\
\text { Mentores } \\
\text { Compañeros } \\
\text { Ingeniero en prácticas }\end{array}$ \\
\hline Examen escrito & $\begin{array}{l}\text { Habilidades basadas en conocimiento } \\
\text { Habilidades numéricas }\end{array}$ & Tutores \\
\hline
\end{tabular}

Tabla 4. Métodos de evaluación en la metodología PBLE. 
Aunque la valoración de cada trabajo depende de cada caso, a título orientativo se proponen los siguientes criterios:

- Valoración del proyecto: 40\%

- Valoración del trabajo individual participativo por el responsable del proyecto: $20 \%$

- Valoración del aprendizaje en el curso (en todas las asignaturas): 40\%

\section{Fase VII: Aspectos organizativos}

La implantación del sistema PBLE requiere algunos cambios estructurales importantes. Por un lado el proceso de valoración del aprendizaje debe recuperar el carácter integrado, exigiendo una síntesis, mediante juntas de calificación o elementos similares que permitan tomar en consideración el trabajo global del alumno.

También el papel del formador se transforma de un modo bastante significativo. En la fase inicial (etapas I y II) el proceso de aprendizaje es liderado por el docente, pero siempre enfocado al proyecto que se está desarrollando. En estas etapas el alumno de las mismas tiene dos referentes de aprendizaje, por un lado su director de proyecto y el proyecto en sí mismo, y por otro lado los docentes en las materias específicas de esas etapas.

Por el contrario en las etapas III y especialmente IV la labor del formador se transforma cada vez más hacia la figura del facilitador o del consultor al servicio del alumno y de su proceso propio de aprendizaje. En efecto en esta etapa el alumno tiene ya capacidad y necesidades específicas para orientar la marcha formativa en sus materias que permiten una optimización a escala individual del mecanismo formativo.

La viabilidad de la implantación de la metodología PBLE desde el punto de vista organizativo del Departamento depende de las posibilidades de financiación que conlleva este proceso. En general, y a la vista de otras experiencias, inicialmente existe un incremento en las necesidades de financiación. No obstante, una vez implantado el sistema pueden conseguirse tasas de autofinanciación interna con origen en proyectos de investigación, con empresas o de innovación educativa. En cualquier caso, el incremento está plenamente justificado si se considera el valor añadido de la formación integral del ingeniero mediante el sistema de aprendizaje con metodología PBLE y su valor diferenciador respecto a otras universidades.

A título de ejemplo algunos puntos que deben considerarse para la elaboración del presupuesto son:

- Plan de sensibilización y formación docente

- Plan de sensibilización y formación de los alumnos

- Proceso de planificación

- Adecuación de espacios

- Recursos de conocimientos: fondos bibliográficos, textos y manuales específicos, artículos técnicos y científicos, normativa, préstamos interbibliotecarios

- Recursos de procesos de fabricación: maquinaria de fabricación, disponibilidades de talleres y laboratorios, métodos de construcción y ensamblaje disponibles, equipos y documentación de seguridad 
METODOLOGÍA PBLE COMO GUÍA DEL PROCESO DE APRENDIZAJE EN INGENIERÍA.

PRIMEROS PASOS EN LA UR.

- Recursos basados en ordenadores: equipos PC, estaciones de trabajo, software, impresoras, redes, manuales

- Recursos basados en pruebas de prestaciones: equipos de medida, hardware de adquisición de datos, recursos para presentaciones, equipos de imagen, audio y video

- Consumibles

\section{CONCLUSIONES}

El núcleo del trabajo especifica y propone una metodología docente integrada basada en el PBLE y bastante revolucionaria en el contexto formativo en ingeniería en España, en particular en la medida en que no se plantea como disociación del proceso formativo entre trabajo en equipo y contenidos teóricos de las asignaturas, sino que se plantea como un elemento integrador del proceso que conlleva indudables mejoras en el proceso de aprendizaje.

Se analiza también la viabilidad de esta metodología docente en los estudios de ingeniería, examinando los profundos cambios que conlleva, tanto en la mentalidad de docentes y alumnos como en la planificación del trabajo, en la evaluación o en la gestión de los recursos.

Esta propuesta está siendo formalizada en términos de alcance, riesgos, medios etc., y puede ser de interés dada la evolución hacia modelos de acreditación de los sistemas formativos de las titulaciones.

Se espera finalizar la formalización de la propuesta a lo largo del curso 2003/2004 para poder comenzar a discutirla en términos de dotación etc., a finales de 2004. El comienzo de la implantación en términos de proyecto piloto se prevé, en el caso de una titulación, en el primer cuatrimestre de 2004/2005. Posteriormente se realizará un diagnóstico basado en indicadores indirectos de proceso, que permita calificar su desarrollo.

\section{BIBLIOGRAFÍA}

ALBANESE, M. y MITCHELL, S. (1993). Problem-based learning: A review of the literature on its outcomes and implementation issues. Academic Medicine, 68 (1), $52-81$

ANGELO, T. y CROSS, P. (1993). Classroom Assessment Techniques: A Handbook for College Teachers. San Francisco: Jossey Bass Publisher.

BANKS, J.C. (1997). Creating \& Assessing Performance-Based Curriculum Projects: A Teacher's Guide to Project-Based Learning \& Performance Assessment. Cats Publications.

BIGGS, J.B. (2002). Aligning Teaching and Assessment to Curriculum Objectives. LTSN Generic Centre at http://www.Itsn.ac.uk

BLUMENFELD, P.C.; SOLOWAY, E.; MARX, R.W.; KRAJCIK, J.S.; GUZDIAL, M. y PALINCSAR, A. (1991). Motivating project-based learning: Sustaining the doing, supporting the learning. Educational Psychologist, 26, 369-398.

CAWLEY, P. (1997). A problem-based module in mechanical engineering, en BOUD, D. \& FELETTI, G. The Challenge of Problem-based Learning. 2nd Edition. London: Kogan Page. 
CROSS, P. (1991). On College Teaching. Journal of Engineering Education, 82 (1), 915.

DELISLE, R. (1997). How to Use Problem-Based Learning in the Classroom. Alexandria: Association for Supervision and Curriculum Development.

DUCH, B. (Ed.) (1995). What is Problem-Based Learning? About Teaching: A Newsletter of the Center for Teaching Effectiveness, 4. En http://www.udel.edu/pbl/cte/jan95-what.html

FARNSWORTH, C.C. (1994). Using computer simulations in problem-based learning. Proceedings of the Thirty-fifth ADCIS Conference, 137-140. Nashville: Omni Press.

HOFFMAN, B. y RITCHIE, D. (1997). Using Multimedia to Overcome the Problems with Problem Based Learning. Instructional Science, 25 (2), 97-115.

HOWARD, K. y SHARP, J. (1996). The Management of a Student Research Project. 2nd Edition. Aldershot: Gower.

LEWIS, V. y HABESHAW, S. (1997). 53 Interesting Ways of Supervising Student Projects. Bristol: Technical and Educational Services.

MCKEACHIE, W. y GIBBS, G. (1999). Teaching Tips: Strategies, Research, and Theory for College and University Teachers. 10th ed. Boston: Houghton Mifflin.

MEHTA, S. y NEM, S. (1998). Computerized Assessment Technique for Large Classes. Journal of Engineering Education, 87 (2), 167-172.

QIN, Z. y JOHNSON, D.W. (1995). Cooperative Versus Competitive Efforts and Problem Solving. Review of Educational Research, 6 (2), 129-143.

STEPIEN, W.J. y GALLAGHER, S.A. (1993). Problem-based Learning: As Authentic as it Gets. Educational Leadership, 50 (7), 25-8.

TOOHEY, S. (1999). Designing Courses for Higher Education. Buckingham: SRHE/Open University Press.

WOODS, D. (1994). Problem-based learning: how to gain the most from PBL. Ontario: Waterdown.

WOODS, D. (1996). Problem-based Learning: resources to gain the most from PBL. En http://www.chemeng.mcmaster.ca/pbl/pbl.htm 